\title{
Risk Factors for Epilepsy in Children With Neonatal Encephalopathy
}

\author{
HANNAH C. GLASS, KAREN J. HONG, ELIZABETH E. ROGERS, RITA J. JEREMY, SONIA L. BONIFACIO, \\ JOSEPH E. SULLIVAN, A. JAMES BARKOVICH, AND DONNA M. FERRIERO
}

\begin{abstract}
Departments of Neurology [H.C.G., J.E.S., D.M.F.], Pediatrics [E.E.R., S.L.B.], Radiology [A.J.B.], Clinical \& Translational Science Institute-Pediatric Clinical Research Center [R.J.J.], University of California at San Francisco, San Francisco, California 94143; University of Washington School of Medicine [K.J.H.], Seattle, Washington 98195
\end{abstract}

\begin{abstract}
We examined neonatal predictors of epilepsy in term newborns with neonatal encephalopathy (NE) by studying children enrolled in a longitudinal, single center cohort study. Clinical data were obtained through chart review, and MRI was performed in the neonatal period. We administered a seizure questionnaire to parents of children aged $\geq 12$ mo (range, 12 mo to $16.5 \mathrm{y}$ ) to determine the outcome of epilepsy. The association between clinical predictors and time to onset of epilepsy was assessed using Cox proportional hazards regression. Thirteen of 129 children developed epilepsy: all had neonatal seizures and brain injury on neonatal MRI. Of the newborns with neonatal seizures, 25\% (15.8/1000 person-years) developed epilepsy, with the highest hazard ratios (HRs) in the newborns with status epilepticus (HR, 35.8; 95\% CI, 6.5-196.5). Children with severe or near-total brain injury were more likely to develop epilepsy compared with those with only mild or moderate injury (HR, 5.5; 95\% CI, 1.8-16.8). In a multivariable analysis adjusting for degree of encephalopathy and severe/near-total brain injury, status epilepticus was independently associated with epilepsy. These data add to information regarding epilepsy pathogenesis and further aid clinicians to counsel parents regarding the likelihood that a newborn with NE will develop epilepsy. (Pediatr Res 70: 535-540, 2011)
\end{abstract}

$\mathrm{T}$ he incidence of neonatal encephalopathy (NE) is about 1 to 2.5 per 1000 live term births, mostly as a result of perinatal asphyxia (1-3). NE is a significant cause of neonatal death and adverse neurodevelopmental outcome such as cerebral palsy, developmental delay, and epilepsy. The reported rate of epilepsy after NE due to hypoxic-ischemic encephalopathy ranges from 9 to $33 \%(4-6)$, and in one study, children with a history of hypoxic-ischemic encephalopathy had five times the risk of developing epilepsy when compared with those without (7).

Although children with NE are known to be at risk for epilepsy, there are few studies examining the neonatal risk factors for developing seizures beyond the newborn period. Past studies in small cohorts have examined severity of encephalopathy, neonatal seizures, and MRI as risk factors with

Received February 18, 2011; accepted May 26, 2011.

Correspondence: Hannah C. Glass, M.D., Department of Neurology, University of California San Francisco, Box 0663, 521 Parnassus Avenue, C-215, San Francisco, CA 94143-0663; e-mail: hannah.glass@ucsf.edu

Supported by the NIH/NCRR UCSF-CTSI Grant Number UL1 RR024131 and NIH/ NINDS grant numbers 5P50NS035902 and NS40117. NIH/NINDS K23NS066137 and the Neonatal Brain Research Institute at UCSF support HCG.

The authors report no conflicts of interest. conflicting results. Pisani et al. (4) found that severe (but not moderate) encephalopathy was associated with later epilepsy, whereas van Kooij et al. (6) found a $10 \%$ prevalence of epilepsy among a cohort of children who suffered moderate encephalopathy. Neonatal seizures were a risk factor in one study, although the effect was not significant after adjusting for the degree of encephalopathy (4). MRI injury is also risk factor for epilepsy $(5,6)$. Small cohort size, inadequate neonatal imaging, or lack of long-term outcome data limit these studies, and counseling for parents of children with NE remains a challenge.

The objective of this study was to evaluate the neonatal clinical and imaging risk factors for childhood epilepsy in a single center cohort of children with a history of NE. All children were imaged using high-resolution MRI according to a standardized research protocol in the newborn period and evaluated longitudinally through childhood.

\section{MATERIALS AND METHODS}

This is a longitudinal cohort study of neonates who were admitted to the Intensive Care Nursery at the University of California San Francisco. Newborns met inclusion criteria of GA $\geq 36 \mathrm{wk}$ at birth and any one of the following: umbilical cord arterial blood $\mathrm{pH}<7.1$, umbilical cord arterial blood base excess $>-10$, or 5-min Apgar score $\leq 5$. These broad inclusion criteria were chosen to encompass newborns with a wide range of injury and neurodevelopmental outcome and have been used in previous publications by our group (8-14). Newborns were excluded if there was evidence of intrauterine or perinatal infection, major anomalies of the brain or other major organ system, or evidence of congenital metabolic disease.

From December 1993 to June 2009, 234 infants $\geq 36$ wk GA at birth were enrolled and imaged according to protocol. Thirty-one children were excluded for the following reasons: deceased during the birth admission (17 children) or withdrew from the study (14 children), leaving 203 subjects.

Clinical data. Trained neonatal research nurses extracted birth delivery data including GA at birth, birth weight, and Apgar scores from medical records. Clinical seizures (spells identified by the attending physician as seizures and treated using anticonvulsant medication), electrographic seizures (events identified by the attending neurophysiologist as seizures), and status epilepticus (recurrent electrographic seizures considered by the neurophysiologist as "status epilepticus," "continuous," or "near continuous" seizures) were determined from chart review. Before 2008, video-EEG monitoring was performed at the discretion of the attending neurologist and neonatologist and performed as standard of care in all children with clinical seizures (minimum duration $30 \mathrm{~min}$ ). Since 2008, video-EEG has been performed for all newborns treated with therapeutic hypothermia from the time of admission until 6-12 $\mathrm{h}$ after rewarming and at least $24 \mathrm{~h}$ after the last recorded EEG seizure. A pediatric neurologist measured the degree of encephalopathy in the first $3 \mathrm{~d}$

Abbreviations: HR, hazard ratio; NE, neonatal encephalopathy; WPPSI, Wechsler Preschool and Primary Scale of Intelligence 
of life on a scale of $0-6$, which considers feeding, alertness, tone, respiratory status, reflexes, and seizures (12).

MRI. All neonates were imaged with MRI using a specialized neonatal head coil on a 1.5-Tesla Signa EchoSpeed system (GE Medical Systems) and using imaging sequences optimized for the neonatal brain $(14,15)$. In cases where more than one MRI was performed during the hospital stay, the MRI closest to day of life 3 to 5 was used for analysis. A pediatric neuroradiologist blinded to the neonatal course prospectively scored the imaging as previously described (8). Injury was scored using a system that is strongly predictive of neurodevelopmental outcome after NE (8). The pattern of injury was described as "basal ganglia/thalamus predominant," "watershed predominant," or "normal" (11). We defined "mild-moderate injury" as a basal ganglia/ thalamus score of 1 or 2 (abnormal signal in the thalamus and/or lentiform nucleus) or watershed score of 1-4 (abnormal signal in the anterior and/or posterior watershed zones), and "severe injury or near-total injury" as a basa ganglia/thalamus score $\geq 3$ or a watershed score of 5 (more extensive involvement in either territory).

Epilepsy interview. The parents of enrolled subjects were contacted by telephone in June 2010, and administered a structured seizure questionnaire that was developed for the purpose of this study. For the children with postneonatal seizures, parents were asked to provide information regarding age of seizure onset, frequency, and medication use. Epilepsy was defined as recurrent, unprovoked seizures, or a single seizure in the setting of abnormal EEG and initiation of medication. For the children who died after hospital discharge, the medical records were reviewed to complete the standardized seizure questionnaire. Epilepsy was graded according to modified Engel classification (class $0=$ seizure free and off seizure medications for at least 6 mo; class $1=$ seizure free for at least 6 mo while on medication or seizure-free off medication for fewer than 6 mo; class $2=$ fewer than one seizure per month on medication; class $3=$ one to four seizures a month on medication; class $4=$ five to thirty seizures per month on medication; class $5=$ thirty or more seizures a month) (16).

Neurodevelopmental follow-up. A pediatric neurologist and a developmental psychologist who were blinded to the neonatal course examined the children at ages 3-6 mo, $1 \mathrm{y}, 2.5 \mathrm{y}, 4 \mathrm{y}$, and $8 \mathrm{y}$. Neuromotor function was evaluated using the neuromotor score, a 5-point scale where 0 is normal and 5 is spastic quadriparesis (8). The neuromotor score was classified as follows: 0 or 1 normal, 2 borderline, $\geq 3$ abnormal. An age-appropriate neuropsychological test was administered to the child at each follow-up appointment. The Bayley Scales of Infant Development were used to test children at $1 \mathrm{y}$ and 2.5 y (Bayley-II before 2008) (17,18). At the age of 4, the Wechsler Preschool and Primary Scale of Intelligence-Revised (WPPSI-R) was used $(19,20)$. The Wechsler Intelligence Scale for Children IV (WISC-IV) (21) was administered for the 8-y follow-up. Scores from standardized tests (all with a mean of 100 and a SD of 15) were used to classify cognitive outcomes as follows: $\geq 85$ on all subscales was considered normal, $70-84$ on one or more subscales was considered borderline, and $<70$ (i.e. 2 SDs below the mean) on one or more subscales was considered abnormal. Comorbidities and most recent neurological and neurodevelopmental examinations were extracted from medical records and the structured seizure questionnaire.

Analysis. Data analyses were conducted using statistical software Stata 10 (StataCorp LP, College Station, TX). The association of clinical predictors with time to onset of epilepsy was assessed using Cox proportional hazards regression and graphically displayed using Kaplan-Meier curves. Data were censored at the time of onset of epilepsy or at the time of the telephone interview in children without epilepsy. Differences between children with and without follow-up were assessed using two-tailed $t$ test for continuous variables, Wilcoxon rank sum for nonparametric data, and $\chi^{2}$ or Fisher's exact test for categorical variables. Multivariable model included those predictors with $p \leq 0.1$ in the univariable analysis. The $p$ values $<0.05$ were considered significant.

The Committee on Human Research at the University of California, San Francisco, approved the protocol. Infants were studied only after informed voluntary parental consent.

\section{RESULTS}

Of the 203 subjects included in the study, we successfully contacted 127 families by telephone and were able to complete adequately adjudicate outcome based on medical records for 2 deceased children, for an overall follow-up rate of $64 \%$. The study subjects are presented in Table 1 . The children whose parents were not available to complete the survey were similar to those whose parents did complete the survey with
Table 1. General clinical characteristics among 129 children with neonatal encephalopathy

Perinatal characteristics

$\begin{array}{lc}\text { Sex, male, } n(\%) & 72(56) \\ \text { GA at birth (wk) } & 39.5( \pm 1.6) \\ \text { Birth weight (g) } & 3331( \pm 617) \\ \text { Cesarean section, } n(\%) & 65(50) \\ \text { 5-min Apgar score*, } n(\%) & \\ \geq 6 & 37(29) \\ 4-5 & 45(35) \\ 0-3 & 46(36) \\ \text { Degree of encephalopathy, } n(\%) & \\ \text { Mild } & 29(22) \\ \text { Moderate } & 41(32) \\ \text { Severe } & 59(46) \\ \text { Therapeutic hypothermia } & 31(24) \\ \text { Neonatal seizures, } n(\%) & \\ \text { No seizures } & 76(59) \\ \text { Clinical only } & 28(22) \\ \text { EEG confirmed seizures } & 19(15) \\ \text { Status epilepticus } & 6(5) \\ \text { MRI injury, } n(\%) & \\ \text { No injury } & 42(33) \\ \text { Mild/moderate } & 65(50) \\ \text { Severe/near-total } & 22(17)\end{array}$

Data are presented as $n(\%)$, mean (SD), or median (range).

* Data missing for one subject.

no differences in sex, GA at birth, birth weight, cord gas $\mathrm{pH}$ or base excess, NE score, and MRI injury scores. However, those whose parents responded were significantly more likely to have had neonatal seizures $(40 \%$ versus $25 \%, p=0.04)$ and have been enrolled in the study more recently (median year of enrollment 2004 versus 1997, $p<0.00005$ ). The median age of surviving study participants at the time of the follow-up survey was 73 mo (6 y; range, 12 mo to $16.5 \mathrm{y}$ ).

Epilepsy features and outcome. Of the 129 evaluated subjects, $13(10 \%)$ developed epilepsy, 7 had febrile seizures, and 1 child had a single spell at age of 2 mo that was not clearly a seizure. The age of epilepsy onset ranged from 0 to $5 \mathrm{y} ; 7$ $(54 \%)$ had onset within the first year of life. Eleven children had abnormal neurodevelopmental outcome, one had borderline outcome and one was not assessed. The clinical characteristics of the 13 study subjects with epilepsy are presented in Table 2.

Perinatal risk factors for epilepsy. Degree of encephalopathy was the only clinical feature associated with epilepsy (Table 3).

Neonatal seizures and risk of epilepsy. All 13 children with epilepsy had a history of clinical and/or electrographic neonatal seizures. The frequency of epilepsy among the 52 children with a history of neonatal seizures was $25 \%$ (or 15.7 per 1000 person years). Among children with neonatal seizures, those with status epilepticus were most likely to develop epilepsy $(5 / 6,83 \%$ or $1237.1 / 1000$ person-years). The single child who had not developed epilepsy was $21 \mathrm{mo}$ at the time of the evaluation and had been treated with therapeutic hypothermia. Those children without status, but whose neonatal seizures EEG confirmed, were more likely to develop epilepsy when compared with those who had only clinical seizures $[5 / 19,26 \%$ or $93.5 / 1000$ person-years versus $3 / 28,11 \%$ or 
Table 2. Clinical seizure, MRI characteristics, and neurodevelopmental outcome for 13 children with neonatal encephalopathy and childhood epilepsy

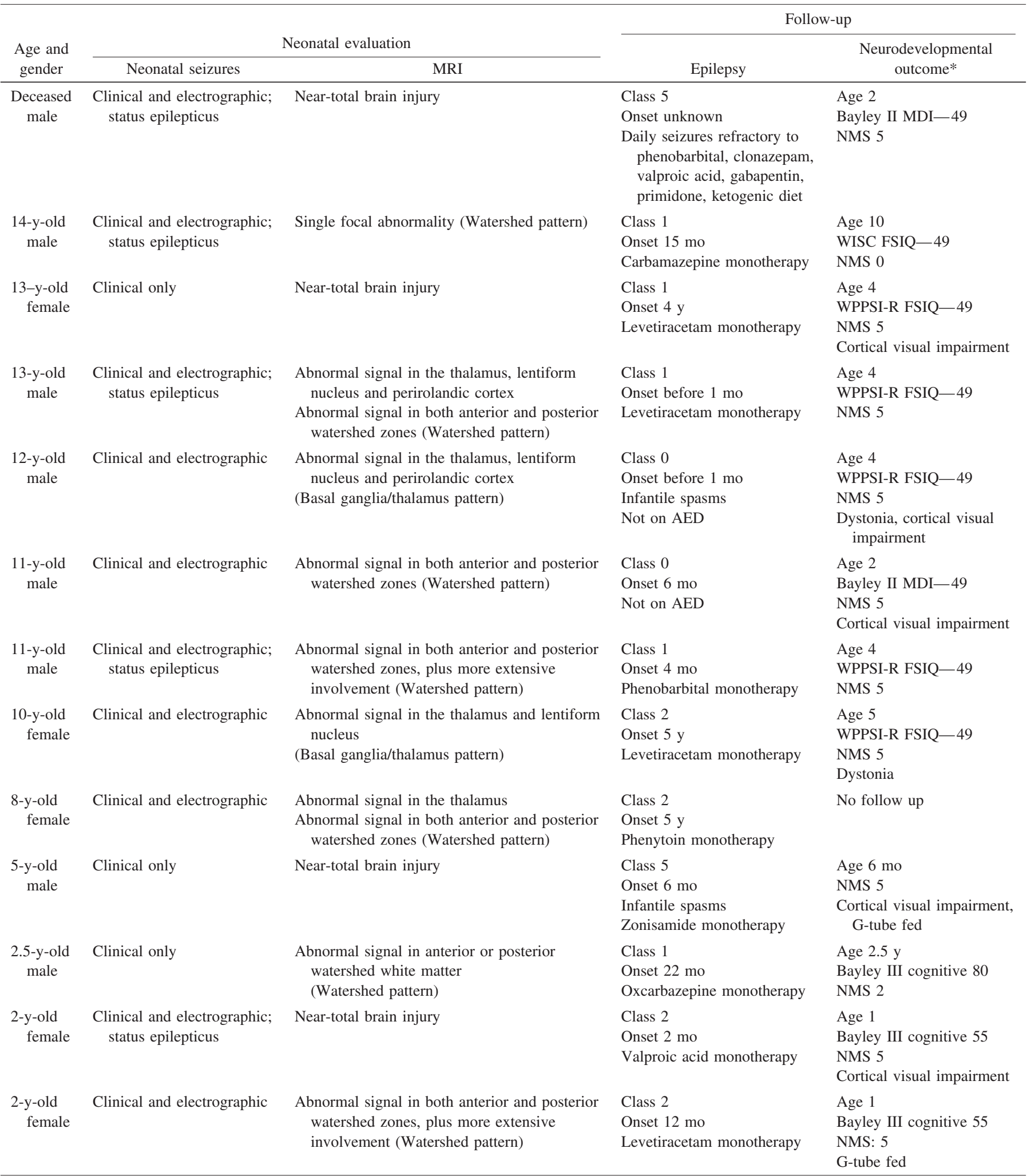

\footnotetext{
* Neurodevelopmental performance: scores are from the last evaluation administered. All tests have a mean of 100 and a standard deviation of 15 . A score of 49 denotes extremely poor performance $>3 \mathrm{SD}$ or lower or untestable.

Bayley II/III, Bayley Scales of Infant Development, second and third edition; MDI, Mental Developmental Index; FSIQ, Full Scale Intelligence Quotient; AED, antiepileptic drug; NMS, neuromotor score; G-tube, gastrostomy tube.
} 
Table 3. Incidence rate and hazard ratios for epilepsy risk factors among 129 children with neonatal encephalopathy

\begin{tabular}{|c|c|c|c|c|c|}
\hline & Person-years & $\begin{array}{l}\text { Epilepsy } \\
\text { cases }\end{array}$ & $\begin{array}{c}\text { Incidence rate/1000 } \\
\text { person-years }(95 \% \mathrm{CI})\end{array}$ & $\operatorname{HR}(95 \% \mathrm{CI})$ & $p$ \\
\hline \multicolumn{6}{|l|}{ Perinatal characteristics } \\
\hline \multicolumn{6}{|l|}{ Sex } \\
\hline Females & 381 & 5 & $13.1(5.5-31.6)$ & - & \\
\hline Males & 444 & 8 & $18.0(9.0-36.1)$ & $1.3(0.4-4.1)$ & 0.6 \\
\hline \multicolumn{6}{|l|}{ Delivery method } \\
\hline Vaginal delivery & 468 & 9 & $19.2(10.0-37.0)$ & - & \\
\hline Cesarean section & 356 & 4 & $11.2(4.2-29.9)$ & $0.5(0.2-1.6)$ & 0.2 \\
\hline \multicolumn{6}{|l|}{ 5-min Apgar score, $n(\%)$} \\
\hline$\geq 6$ & 305 & 2 & $6.6(1.6-26.2)$ & - & \\
\hline $4-5$ & 280 & 6 & $21.5(9.6-47.8)$ & $2.8(0.6-18.8)$ & \\
\hline $0-3$ & 238 & 5 & $21.0(8.7-50.5)$ & $2.5(0.5-12.8)$ & 0.4 \\
\hline \multicolumn{6}{|c|}{ Degree of encephalopathy $(N=100)^{*}$} \\
\hline Moderate & 305 & 1 & $3.3(0.5-23.2)$ & - & \\
\hline Severe & 267 & 12 & $45.0(25.5-79.2)$ & $10.7(1.4-82.7)$ & 0.002 \\
\hline \multicolumn{6}{|l|}{ Therapeutic hypothermia } \\
\hline Not treated & 774 & 11 & $14.2(7.9-25.7)$ & & \\
\hline Treated & 51 & 2 & $39.4(9.9-157.6)$ & $0.9(0.2-4.1)$ & 0.8 \\
\hline \multicolumn{6}{|l|}{ Neonatal seizures $(N=53) \dagger$} \\
\hline Clinical only & 206 & 3 & $14.6(4.7-45.1)$ & - & \\
\hline EEG confirmed seizures & 53 & 5 & $93.5(38.9-224.7)$ & $4.0(0.9-17.3)$ & \\
\hline Status epilepticus & 4 & 5 & $1237.1(514.9-2972.2)$ & $35.8(6.5-196.5)$ & 0.0001 \\
\hline \multicolumn{6}{|l|}{ MRI injury $(N=87) \ddagger$} \\
\hline Mild/moderate & 434 & 5 & $11.5(4.8-27.7)$ & - & \\
\hline Severe/near-total & 146 & 8 & $54.7(27.4-109.4)$ & $5.5(1.8-16.8)$ & 0.003 \\
\hline
\end{tabular}

* No cases of epilepsy among children with mild neonatal encephalopathy.

$\dagger$ No cases of epilepsy among children without neonatal seizures.

\$ No cases of epilepsy among children without injury on neonatal MRI.

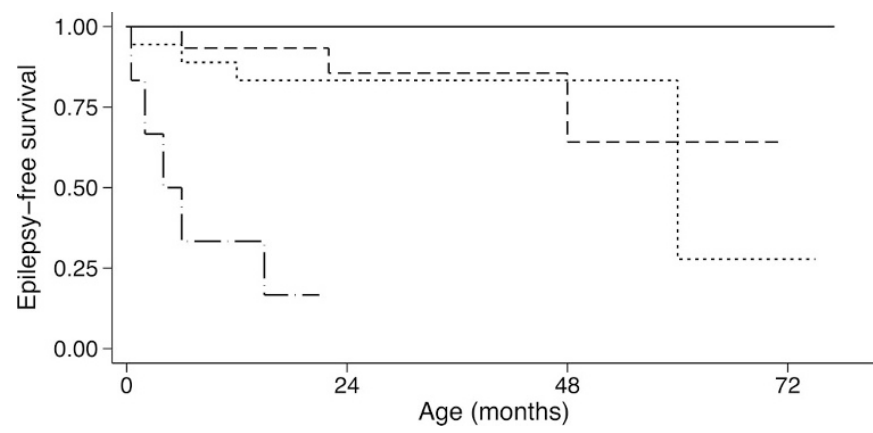

Figure 1. Kaplan-Meier estimates for epilepsy-free survival among 129 newborns with NE, with and without neonatal seizures. No seizures, solid line $(n=76)$; clinical seizures only, dashed line $(n=28)$; EEG confirmed seizures, dotted line $(n=19)$; status epilepticus, dash-dot line $(n=6)$.

14.5/1000 person-years; hazard ratio (HR), 4.0; 95\% CI, $0.9-17.3]$. The epilepsy-free survival data in those children with and without a history of neonatal seizures are presented in Figure 1.

Brain injury and epilepsy. All children with epilepsy had injury on neonatal MRI, and the severity of injury was associated with epilepsy. The children with severe or near-total brain injury were more likely to develop epilepsy $(8 / 22,36 \%$ or $54.7 / 1000$ person-years) versus $5 / 65$ (8\% or $11.5 / 1000$ person-years) for those with mild or moderate injury. Of the 16 children with severe or near-total brain injury and neonatal seizures, half developed epilepsy. Pattern of injury was also important: of the 30 children with basal ganglia/thalamus predominant pattern of injury, six (20\% or 32.3/1000 personyears) developed epilepsy, whereas only seven $(12 \%, 17.7 /$ 1000 person years) with watershed predominant pattern of

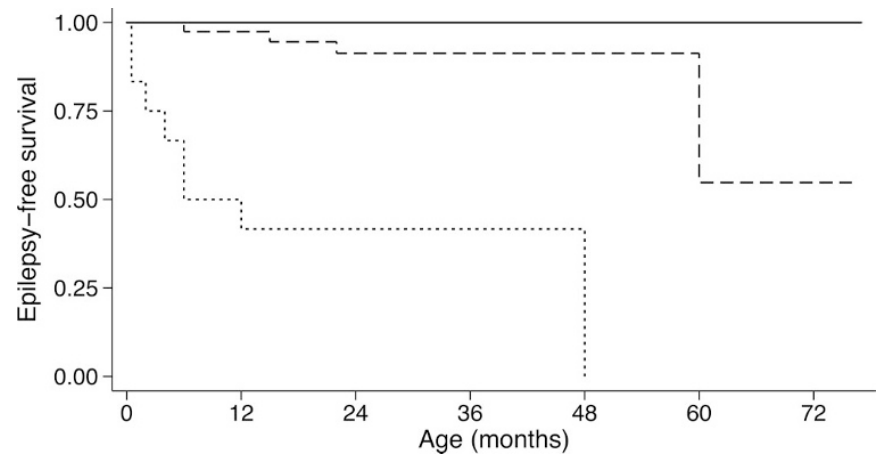

Figure 2. Kaplan-Meier estimates for epilepsy-free survival among 129 newborns with NE, with and without brain injury. No injury, solid line ( $n=$ 42); mild/moderate injury, dashed line $(n=65)$; severe/near-total injury, dotted line $(n=22)$.

injury developed epilepsy $(p=0.005)$. When compared with those children with mild/moderate brain injury, those with severe or near-total injury had a higher rate of epilepsy (HR, 5.5; 95\% CI, 1.8-16.8; $p=0.003$ ). Epilepsy-free survival by severity of brain injury is presented in Figure 2.

Adjusted analysis. In a multivariable analysis among those with moderate or severe encephalopathy, neonatal seizures and MRI injury, adjusting for NE, status epilepticus remained a highly significant risk factor for epilepsy (HR, 17.3; 95\% CI, $2.7-110.0 ; p=0.003$ ), whereas severe/near-total injury (HR, 2.4; 95\% CI, 0.7-8.4; $p=0.2$ ) was not (Table 4).

\section{DISCUSSION}

In this longitudinal sample of 129 newborns with NE, neonatal seizures and brain injury on MRI were strong risk 
Table 4. Adjusted risk of epilepsy among those newborns with MRI injury, neonatal seizures, and moderate or severe neonatal encephalopathy $(\mathrm{N}=42)$

\begin{tabular}{lcl}
\hline & $\begin{array}{c}\text { Adjusted HR } \\
(95 \% \mathrm{CI})\end{array}$ & \multicolumn{1}{c}{$p$} \\
\hline Severe encephalopathy & $4.5(0.5-40.6)$ & 0.2 \\
Severe/near-total brain injury & $2.4(0.7-8.4)$ & 0.2 \\
EEG confirmed seizures & $4.1(0.8-21.0)$ & 0.09 \\
Status epilepticus & $17.3(2.7-110.0)$ & 0.003 \\
\hline
\end{tabular}

factors for epilepsy, and the children with epilepsy all had adverse neurodevelopmental outcome.

These results support previous studies suggesting that epilepsy occurs in children with a more severe spectrum of NE. The $25 \%$ frequency of epilepsy after neonatal seizures is similar to previous contemporary cohorts that used broad clinical and/or electrographic definitions for neonatal seizures and lower than studies that required EEG confirmation of seizures (22-25). Although duration of follow-up is variable across studies, highest risk within the first year of life is a common feature, as is the high rate of associated neurodevelopmental disabilities $(23,25-28)$.

Animal studies have begun to elucidate mechanisms by which neonatal seizures can induce persistent enhanced neocortical excitability (29). Our data also support evidence that severity of seizures is important, which is in keeping with studies by Clancy and Legido (22), who showed in a mixed cohort that a higher burden of neonatal seizures was a risk factor for epilepsy, and Pisani et al., (30) who showed a higher rate of epilepsy after status epilepticus when compared with recurrent seizures.

Severity and pattern of MRI injury are known risk factors for neonatal seizures $(6,11)$, and we also show a clear relationship with epilepsy. Children with severe basal ganglia/ thalamus pattern of injury and cortical involvement were at highest risk of developing epilepsy. There is controversy from animal studies regarding whether neuronal injury is required for acquired epileptogenesis in the immature brain, or whether seizures alone without neuronal injury can cause changes in excitability sufficient to result in unprovoked seizures beyond the neonatal period (31). Our finding, that epilepsy occurred only in those children with apparent brain injury on MRI, supports, but does not prove, the hypothesis that neuronal injury is, in fact, required to develop epilepsy.

Although our findings do not support a reduced risk of epilepsy in the cooled subjects, the data we present do not exclude this possibility. Since fall 2007, we have treated most newborns at risk for hypoxic-ischemic injury and moderatesevere encephalopathy with therapeutic hypothermia using whole body cooling. Therapeutic hypothermia, which has been shown to reduce death and disability at $18-22$ mo in several randomized, controlled trials (32-35) also has an antiseizure effect in animal models $(36,37)$, although its effect on seizures in human newborns is not clear. Among newborns treated with therapeutic hypothermia at our center, electrographic seizures are present in $>30 \%$. If neonatal seizures are suppressed in human newborns treated with hypothermia and neonatal seizures enhance long-term epileptogenesis, the risk of epilepsy in the cooled population may ultimately be reduced. The question of whether hypothermia reduces neonatal seizure burden and/or epileptogenesis should be further explored in larger data sets.

Although this is a large cohort with high-quality neonatal imaging and long-term follow-up, the data are limited in several ways. First, the survey response rate was only $64 \%$. This limitation may have led to an overestimate of the prevalence of epilepsy, because the rate of neonatal seizures was higher in those children whose parents responded to the interview. However, response rate should not affect the relationships between perinatal risk factors, including seizures and MRI findings, and epilepsy. Second, it is possible that there was bias toward diagnosis of epilepsy in some cases of children with ambiguous spells and severe developmental impairment. This may have falsely increased the relationship between MRI injury and neonatal seizures and epilepsy. Third, since the study's inception in 1993, we have changed the guidelines for monitoring and treating newborns with encephalopathy. Before 2008, video-EEG was used at the discretion of the attending neurologist, usually for 30-60 min routine recordings. Since 2008, we have monitored all children with NE using continuous video-EEG for the duration of hypothermia and rewarming. To understand the true relationship between clinical versus electrographic seizures and epilepsy, we will need to examine a larger cohort of subjects with long-term conventional video-EEG monitoring.

\section{CONCLUSIONS}

Epilepsy is an outcome following brain injury and neonatal seizures in the setting of severe hypoxic-ischemic brain injury. These data add to our understanding of the pathogenesis of epilepsy after seizures in the newborn period and provide information for clinicians and parents planning long-term care for children with NE. For term infants with NE, but without both neonatal seizures and brain injury on MRI, parents can be reassured that the child is unlikely to develop epilepsy. Children with electrographic seizures (especially status epilepticus) and severe brain injury have a high risk of adverse neurodevelopmental outcome, including epilepsy. This highrisk population will be important for future studies examining neuroprotective and antiepileptic agents. Neonatal seizures and status epilepticus are potentially modifiable risk factors, and there is urgent need for studies to examine whether early monitoring and treatment will improve outcome.

Acknowledgments. We thank Dr. Charles E. McCullough, the study nurses from the NCRC, project coordinators Veronica de Santiago, and Laurel Haeusslein, as well as the participants of the KL2 Works in Progress program. Amy Markowitz provided editorial support.

\section{REFERENCES}

1. Thornberg E, Thiringer K, Odeback A, Milsom I 1995 Birth asphyxia: incidence, clinical course and outcome in a Swedish population. Acta Paediatr 84:927-932

2. Graham EM, Ruis KA, Hartman AL, Northington FJ, Fox HE 2008 A systematic review of the role of intrapartum hypoxia-ischemia in the causation of neonatal encephalopathy. Am J Obstet Gynecol 199:587-595 
3. Kurinczuk JJ, White-Koning M, Badawi N 2010 Epidemiology of neonatal encephalopathy and hypoxic-ischaemic encephalopathy. Early Hum Dev 86:329-338

4. Pisani F, Orsini M, Braibanti S, Copioli C, Sisti L, Turco EC 2009 Development of epilepsy in newborns with moderate hypoxic-ischemic encephalopathy and neonatal seizures. Brain Dev 31:64-68

5. Belet N, Belet U, Incesu L, Uysal S, Ozinal S, Keskin T, Sunter AT, Kucukoduk S 2004 Hypoxic-ischemic encephalopathy: correlation of serial MRI and outcome Pediatr Neurol 31:267-274

6. van Kooij BJ, van Handel M, Nievelstein RA, Groenendaal F, Jongmans MJ, de Vries LS 2010 Serial MRI and neurodevelopmental outcome in 9- to 10-year-old children with neonatal encephalopathy. J Pediatr 157:221.e2-e227.e2

7. Bergamasco B, Benna P, Ferrero P, Gavinelli R 1984 Neonatal hypoxia and epileptic risk: a clinical prospective study. Epilepsia 25:131-136

8. Barkovich AJ, Hajnal BL, Vigneron D, Sola A, Partridge JC, Allen F, Ferriero DM 1998 Prediction of neuromotor outcome in perinatal asphyxia: evaluation of MR scoring systems. AJNR Am J Neuroradiol 19:143-149

9. Miller SP, Newton N, Ferriero DM, Partridge JC, Glidden DV, Barnwell A, Chuang NA, Vigneron DB, Barkovich AJ 2002 Predictors of 30-month outcome after perinatal depression: role of proton MRS and socioeconomic factors. Pediatr Res 52:71-77

10. Miller SP, Weiss J, Barnwell A, Ferriero DM, Latal-Hajnal B, Ferrer-Rogers A, Newton N, Partridge JC, Glidden DV, Vigneron DB, Barkovich AJ 2002 Seizureassociated brain injury in term newborns with perinatal asphyxia. Neurology 58:542-548

11. Miller SP, Ramaswamy V, Michelson D, Barkovich AJ, Holshouser B, Wycliffe N, Glidden DV, Deming D, Partridge JC, Wu YW, Ashwal S, Ferriero DM 2005 Patterns of brain injury in term neonatal encephalopathy. J Pediatr 146:453-460

12. Miller SP, Latal B, Clark H, Barnwell A, Glidden D, Barkovich AJ, Ferriero DM, Partridge JC 2004 Clinical signs predict 30-month neurodevelopmental outcome after neonatal encephalopathy. Am J Obstet Gynecol 190:93-99

13. Hajnal BL, Sahebkar-Moghaddam F, Barnwell AJ, Barkovich AJ, Ferriero DM 1999 Early prediction of neurologic outcome after perinatal depression. Pediatr Neurol 21:788-793

14. Barkovich AJ, Miller SP, Bartha A, Newton N, Hamrick SE, Mukherjee P, Glenn $\mathrm{OA}, \mathrm{Xu}$ D, Partridge JC, Ferriero DM, Vigneron DB 2006 MR imaging, MR spectroscopy, and diffusion tensor imaging of sequential studies in neonates with encephalopathy. AJNR Am J Neuroradiol 27:533-547

15. Bonifacio SL, Glass HC, Vanderpluym J, Agrawal AT, Xu D, Barkovich AJ, Ferriero DM 2011 Perinatal events and early magnetic resonance imaging in therapeutic hypothermia. J Pediatr 158:360-365

16. Golomb MR, Garg BP, Carvalho KS, Johnson CS, Williams LS 2007 Perinatal stroke and the risk of developing childhood epilepsy. J Pediatr 151:409-413, 413 e1-e2

17. Bayley N 1993 Bayley Scales of Infant Development-Second Edition (BSID-II) Manual. The Psychological Corporation, San Antonio, pp 227

18. Bayley N 2005 Bayley Scales of Infant and Toddler Development-Third Edition (Bayley-III) Technical Manual. The Psychological Corporation, San Antonio, pp 109

19. Wechsler D 1989 Wechsler Preschool and Primary Scale of Intelligence-Revised (WPPSI-R) Administration Manual. The Psychological Corporation, San Antonio, pp 123
20. Wechsler D 2002 Wechsler Preschool and Primary Scale of Intelligence-Third Edition (WPPSI-III) Technical Manual. The Psychological Corporation, San Antonio, pp 130

21. Wechsler D 2003 Wechsler Intelligence Scale for Children-Fourth Edition (WISCIV) Integrated Technical and Interpretive Manual. The Psychological Corporation, San Antonio, pp 100

22. Clancy RR, Legido A 1991 Postnatal epilepsy after EEG-confirmed neonatal seizures. Epilepsia 32:69-76

23. Ronen GM, Buckley D, Penney S, Streiner DL 2007 Long-term prognosis in children with neonatal seizures: a population-based study. Neurology 69:1816-1822

24. Guillet R, Kwon J 2007 Seizure recurrence and developmental disabilities after neonatal seizures: outcomes are unrelated to use of phenobarbital prophylaxis. J Child Neurol 22:389-395

25. Legido A, Clancy RR, Berman PH 1991 Neurologic outcome after electroencephalographically proven neonatal seizures. Pediatrics 88:583-596

26. McBride MC, Laroia N, Guillet R 2000 Electrographic seizures in neonates correlate with poor neurodevelopmental outcome. Neurology 55:506-513

27. Brunquell PJ, Glennon CM, DiMario FJ Jr, Lerer T, Eisenfeld L 2002 Prediction of outcome based on clinical seizure type in newborn infants. J Pediatr 140:707-712

28. Glass HC, Glidden D, Jeremy RJ, Barkovich AJ, Ferriero DM, Miller SP 2009 Clinical neonatal seizures are independently associated with outcome in infants at risk for hypoxic-ischemic brain injury. J Pediatr 155:318-323

29. Isaeva E, Isaev D, Savrasova A, Khazipov R, Holmes GL 2010 Recurrent neonatal seizures result in long-term increases in neuronal network excitability in the rat neocortex. Eur J Neurosci 31:1446-1455

30. Pisani F, Cerminara C, Fusco C, Sisti L 2007 Neonatal status epilepticus vs recurrent neonatal seizures: clinical findings and outcome. Neurology 69:2177-2185

31. Dudek FE, Ekstrand JJ, Staley KJ 2010 Is neuronal death necessary for acquired epileptogenesis in the immature brain? Epilepsy Curr 10:95-99

32. Shankaran S, Laptook AR, Ehrenkranz RA, Tyson JE, McDonald SA, Donovan EF, Fanaroff AA, Poole WK, Wright LL, Higgins RD, Finer NN, Carlo WA, Duara S, Oh W, Cotten CM, Stevenson DK, Stoll BJ, Lemons JA, Guillet R, Jobe AH 2005 Whole-body hypothermia for neonates with hypoxic-ischemic encephalopathy. N Engl J Med 353:1574-1584

33. Azzopardi DV, Strohm B, Edwards AD, Dyet L, Halliday HL, Juszczak E, Kapellou O, Levene M, Marlow N, Porter E, Thoresen M, Whitelaw A, Brocklehurst P 2009 Moderate hypothermia to treat perinatal asphyxial encephalopathy. N Engl J Med $361: 1349-1358$

34. Gluckman PD, Wyatt JS, Azzopardi D, Ballard R, Edwards AD, Ferriero DM, Polin RA, Robertson CM, Thoresen M, Whitelaw A, Gunn AJ 2005 Selective head cooling with mild systemic hypothermia after neonatal encephalopathy: multicentre randomised trial. Lancet 365:663-670

35. Edwards AD, Brocklehurst P, Gunn AJ, Halliday H, Juszczak E, Levene M, Strohm B, Thoresen M, Whitelaw A, Azzopardi D 2010 Neurological outcomes at 18 months of age after moderate hypothermia for perinatal hypoxic ischaemic encephalopathy: synthesis and meta-analysis of trial data. BMJ 340:c363

36. Liu CQ, Xia YF, Yuan YX, Li L, Qiu XL 2010 [Effects of selective head cooling with mild hypothermia on serum levels of caspase- 3 and IL-18 in neonates with hypoxic-ischemic encephalopathy]. Zhongguo Dang Dai Er Ke Za Zhi 12:690-692

37. Wang Y, Liu PP, Li LY, Zhang HM, Li T 2010 Hypothermia reduces brain edema, spontaneous recurrent seizure attack, and learning memory deficits in the kainic acid treated rats. CNS Neurosci Ther [Epub ahead of print] 